\title{
Immunostaining for peroxisome proliferator gamma distinguishes dedifferentiated liposarcoma from other retroperitoneal sarcomas
}

\author{
Andrew E Horvai ${ }^{1}$, Jochen T Schaefer ${ }^{1}$, Eric K Nakakura ${ }^{2}$ and Richard J O’Donnell ${ }^{3}$ \\ ${ }^{1}$ Department of Pathology, University of California, San Francisco, CA, USA; ${ }^{2}$ Department of Surgery, \\ University of California, San Francisco, CA, USA and ${ }^{3}$ Department of Orthopaedic Surgery, University \\ of California, San Francisco, CA, USA
}

\begin{abstract}
Dedifferentiated liposarcoma can be readily diagnosed by the juxtaposition of a well-differentiated liposarcoma to a nonlipogenic sarcoma. However, if the lipogenic component is not abundant due to surgical sampling or small biopsy, dedifferentiated liposarcoma can be difficult to distinguish from other poorly different sarcomas. Peroxisome proliferator-activated receptor gamma (PPAR- $\gamma$ ) is a nuclear hormone receptor that plays a critical role in adipocyte differentiation. Prior studies have not only demonstrated PPAR- $\gamma$ mRNA in various subtypes of liposarcoma but have also shown that adipocyte differentiation can be induced in some liposarcomas by a PPAR- $\gamma$ agonist. In the present study, we investigated whether immunostaining for PPAR- $\gamma$ can be used to distinguish dedifferentiated liposarcoma from other retroperitoneal sarcomas. We examined a series of 40 dedifferentiated liposarcoma and compared the staining for PPAR- $\gamma$ to a series of 24 retroperitoneal sarcomas that lacked lipogenic differentiation. A monoclonal antibody against PPAR- $\gamma$ was used to stain formalin-fixed paraffin-embedded tissue. Specific nuclear immunostaining was present in $37 / 40(93 \%)$ of the dedifferentiated liposarcoma and $6 / 24(25 \%)$ of the other sarcomas (two leiomyosarcomas and four undifferentiated sarcomas). Interestingly, immunostaining for CDK4 and/or MDM2 was identified in three of the four PPAR- $\gamma$-positive undifferentiated sarcomas, raising the possibility that these may represent dedifferentiated liposarcoma. This is the first study demonstrating the utility of PPAR- $\gamma$ immunohistochemistry in the diagnosis of dedifferentiated liposarcoma in tissue sections. Although not completely specific, the presence of PPAR- $\gamma$ staining, in combination with histologic findings and other markers, can aid in the diagnosis of dedifferentiated liposarcoma, particularly on small biopsies that may not sample the well-differentiated component.

Modern Pathology (2008) 21, 517-524; doi:10.1038/modpathol.3801017; published online 18 January 2008
\end{abstract}

Keywords: dedifferentiated liposarcoma; undifferentiated sarcoma; retroperitoneum; PPAR- $\gamma$; immunohistochemistry

Liposarcoma is one of the most common soft tissue sarcomas in adults. ${ }^{1}$ The liposarcomas can be divided into three major categories: the well-differentiated-dedifferentiated subtype, myxoid round cell subtype, and pleomorphic subtype. Dedifferentiated liposarcoma is defined as a tumor composed of both a well-differentiated liposarcoma and a second sarcoma without lipogenic differentiation. The latter frequently presents as an undifferentiated

Correspondence: Dr AE Horvai, MD, PhD, Department of Pathology, University of California, 1600 Divisadero Drive B220, San Francisco, CA 94115-1656, USA.

E-mail: andho@itsa.ucsf.edu

Received 15 October 2007; revised and accepted 04 December 2007; published online 18 January 2008 sarcoma or a so-called malignant fibrous histiocytoma. $^{1,2}$ Whether the nonlipogenic component represents true 'dedifferentiation' remains controversial. For practical purposes, the term dedifferentiated liposarcoma characterizes a distinct clinicopathologic entity with two histologic components. ${ }^{1}$ Dedifferentiated liposarcoma most commonly presents as the 'primary' form in which both components are identified simultaneously. In the more rare 'secondary' form, the dedifferentiated component presents as a recurrence, sometimes years after the initial diagnosis of well-differentiated liposarcoma. ${ }^{3}$ Dedifferentiated liposarcoma is most commonly located in the retroperitoneum and, unlike well-differentiated liposarcoma, exhibits metastatic potential. ${ }^{1}$ 
In the appropriate clinical context, histologic identification of well-differentiated liposarcoma closely associated with a second sarcoma is usually sufficient for the diagnosis of dedifferentiated liposarcoma. However, the presence of a spindle cell or pleomorphic sarcoma of the retroperitoneum can be a diagnostic challenge when the surrounding adipose tissue is not available for histologic analysis, a common problem in small biopsies. Two additional factors make the diagnosis of dedifferentiated liposarcoma problematic. First, a growing body of evidence suggests that a subset of poorly differentiated sarcomas of the retroperitoneum, formerly classified as myxofibrosarcoma or so-called 'malignant fibrous histiocytoma', actually represents dedifferentiated liposarcoma. ${ }^{4,5}$ Second, the prognosis of dedifferentiated liposarcoma is largely independent of the grade of the dedifferentiated component such that even histologically lowgrade, fibromatosis-like examples have metastatic potential. $^{3}$ Nevertheless, some studies have suggested that dedifferentiated liposarcoma carries a more favorable prognosis than other retroperitoneal sarcomas, such as leiomyosarcoma or so-called malignant fibrous histiocytoma (although this difference in outcome is the subject of some debate). ${ }^{3,6}$ Therefore, diagnostic markers that reliably distinguish dedifferentiated liposarcoma from other retroperitoneal sarcomas have the potential for significant practical utility.

Recent evidence suggests that the detection of overexpression of the cell cycle regulators $M d m 2$ and Cdk4 is useful in the diagnosis of liposarcoma. ${ }^{7,8}$ For example, the immunohistochemical detection of MDM2 and CDK4 proteins is useful to distinguish well-differentiated liposarcoma from benign lipoma. However, MDM2 and CDK4 staining is not exclusive to dedifferentiated liposarcoma, as other sarcomas also stain for these markers. ${ }^{8-11}$ The latter result is perhaps not surprising, given that disparate malignant tumors can share abnormalities in cell-cycle control, whereas such abnormalities are uncommon in benign tumors (such as lipoma) with more limited growth potential.

Peroxisome proliferator-activated receptor gamma (PPAR- $\gamma)$ is a nuclear hormone receptor that plays a critical role in adipocyte differentiation. PPAR- $\gamma$ heterodimerizes with the retinoid X receptor family of steroid hormone receptors and promotes transcription at specific DNA motifs termed PPARresponse elements at adipocyte-specific genes. ${ }^{12-14}$ Prior studies have demonstrated PPAR $-\gamma$ mRNA in various subtypes of liposarcoma but not other sarcomas. Further, terminal adipocyte differentiation can be induced in myxoid/round cell and welldifferentiated liposarcomas in vitro ${ }^{15}$ by thiazolidenediones, a class of drugs that activate PPAR- $\gamma$. Therapy targeted against PPAR- $\gamma$ to induce liposarcoma differentiation and growth met with mixed success in early clinical trials. ${ }^{16,17}$ Taken together, these data suggest that liposarcoma cells may retain the differentiation program controlled by PPAR $-\gamma$ in some tumors, whereas in others the program may be blocked during transformation or progression. Unlike MDM2 and CDK4, PPAR- $\gamma$ is abundantly expressed in benign adipocytes, so the presence of PPAR- $\gamma$ does not distinguish between benign and malignant adipose tumors. However, if expression of PPAR $-\gamma$ persists in the dedifferentiated component of dedifferentiated liposarcoma, but not in morphologic mimics, it may serve as a useful adjunct in the diagnosis of difficult cases.

The goal of this study was to examine the extent of PPAR- $\gamma$ expression as detected immunohistochemically in both well-differentiated and dedifferentiated components of dedifferentiated liposarcoma and to test the diagnostic utility of PPAR- $\gamma$ immunostaining in distinguishing dedifferentiated liposarcoma from other types of retroperitoneal sarcomas. We employed immunohistochemical staining for PPAR- $\gamma$ expression in paraffin-embedded sections of dedifferentiated liposarcoma and compared the results with other retroperitoneal sarcomas that had no histologic evidence of lipogenic differentiation.

\section{Materials and methods}

\section{Selection of Cases}

Forty consecutive cases of dedifferentiated liposarcoma were identified from the Department of Pathology files of the University of California, San Francisco. The diagnosis was based on light microscopic, radiographic, and clinical features. The 'gold standard' of diagnosis in every case was the presence of well-differentiated liposarcoma, either adjacent to macroscopic areas of sarcoma without lipogenic differentiation (primary) or previously removed from the same anatomic site (secondary). The grade of the dedifferentiated component was recorded based on previously published criteria. ${ }^{2,18}$ A second group of 24 consecutive retroperitoneal sarcomas, for which dedifferentiated liposarcoma might be considered in the differential diagnosis based on size, histology, and location, was identified. Based on morphology and immunophenotype, cases were classified according to the latest WHO classification of bone and soft tissue tumors. ${ }^{1}$ Only those sarcomas that lacked evidence of a well-differentiated liposarcoma component by careful examination of surrounding adipose tissue were included.

\section{Immunohistochemistry}

Immunohistochemical analysis was performed using previously published techniques ${ }^{19}$ on archival, paraffin-embedded sections. Briefly, $4 \mu \mathrm{m}$ paraffin-embedded sections were deparaffinized, heated in EDTA buffer (Cell Marque, Rocklin, CA, 
USA), blocked, and incubated with a monoclonal antibody to PPAR- $\gamma$ (E-8; Santa Cruz Biotechnology, Santa Cruz, CA, USA) at 1:50 dilution, CDK4 (DCS31, Biosource, Camarillo, CA, USA) at 1:100 dilution, or MDM2 (IF2, Zymed, South San Francisco, CA, USA) at 1:100 dilution. Detection of all antibodies used the Envision system (DAKO, Carpinteria, CA, USA) according to the manufacturer's directions. The primary antibody dilution for PPAR- $\gamma$ was based on optimization using normal adipose tissue. Slides were scored as positive if more than $10 \%$ of tumor cells showed specific, strong, nuclear staining. Positive cases were stratified as follows: ${ }^{20}$

level 1: $>0 \%$ but $<25 \%$ positive cells;

level 2: $>25 \%$ but $<50 \%$ positive cells; level 3: $>50 \%$ but $<90 \%$ positive cells; level 4: $>90 \%$ positive cells.

\section{Results}

The morphologic, clinical, and immunophenotypic characteristics of the dedifferentiated liposarcomas are summarized in Table 1 . The majority of dedifferentiated liposarcoma were located in the retroperitoneum $(24 / 40,60 \%)$, were high grade $(32 / 40$, $80 \%)$, and were primary tumors $(34 / 40,85 \%)$, denoting that the well-differentiated and dedifferentiated components were present in the initial resection specimen. Figure 1 illustrates representative examples of hematoxylin and eosin (H\&E)stained, well-differentiated (Figure 1a), high-grade dedifferentiated (Figure 1b), and low-grade dedifferentiated (Figure 1c) components. Two cases showed heterologous osteosarcomatous differentiation and one case showed the previously described meningothelial-like differentiation. ${ }^{21,22}$ Specific nuclear staining with PPAR- $\gamma$ was identified in $35 / 40(88 \%)$ well-differentiated components and

Table 1 Summary of clinical, pathologic, and immunohistochemical data for dedifferentiated liposarcomas

\begin{tabular}{|c|c|c|c|c|c|}
\hline \multirow[t]{2}{*}{ Case } & \multirow[t]{2}{*}{ Site } & \multirow[t]{2}{*}{ Grade } & \multirow[t]{2}{*}{ Presentation } & \multicolumn{2}{|c|}{ PPAR- $\gamma$ immunohistochemistry } \\
\hline & & & & $W D$ & $D D$ \\
\hline 1 & Retroperitoneum & Low & Primary & 2 & 1 \\
\hline 2 & Small intestine & Low & Secondary & 2 & 1 \\
\hline 3 & Retroperitoneum & High & Secondary & 1 & 1 \\
\hline 4 & Retroperitoneum & Low & Primary & 1 & 1 \\
\hline 5 & Retroperitoneum & High & Primary & 2 & 1 \\
\hline 6 & Trunk & High & Primary & 1 & 1 \\
\hline 7 & Thigh & High & Primary & 1 & 0 \\
\hline 8 & Retroperitoneum & High & Primary & 2 & 3 \\
\hline 9 & Retroperitoneum & High & Secondary & 2 & 1 \\
\hline 10 & Right thigh & High & Primary & 1 & 3 \\
\hline 11 & Gluteal & High & Primary & 2 & 3 \\
\hline 12 & Retroperitoneum & High & Primary & 1 & 2 \\
\hline 13 & Arm & High & Primary & 1 & 2 \\
\hline 14 & Spermatic cord & High & Primary & 2 & 1 \\
\hline 15 & Thigh & High & Primary & 0 & 0 \\
\hline 16 & Spermatic cord & Low & Primary & 2 & 3 \\
\hline 17 & Retroperitoneum & High & Primary & 1 & 1 \\
\hline 18 & Retroperitoneum & High & Primary & 1 & 3 \\
\hline 19 & Retroperitoneum & High & Primary & 1 & 2 \\
\hline 20 & Arm & High & Primary & 2 & 3 \\
\hline 21 & Trunk & High & Primary & 0 & 3 \\
\hline 22 & Thigh & Low & Primary & 0 & 3 \\
\hline 23 & Retroperitoneum & High & Primary & 2 & 3 \\
\hline 24 & Thigh & High & Primary & 2 & 3 \\
\hline 25 & Retroperitoneum & High & Secondary & 3 & 3 \\
\hline 26 & Retroperitoneum & Low & Primary & 1 & 1 \\
\hline 27 & Retroperitoneum & High & Primary & 1 & 1 \\
\hline 28 & Retroperitoneum & High & Secondary & 1 & 3 \\
\hline 29 & Trunk & High & Primary & 2 & 3 \\
\hline 30 & Retroperitoneum & High & Primary & 1 & 3 \\
\hline 31 & Spermatic cord & Low & Primary & 0 & 1 \\
\hline 32 & Retroperitoneum & High & Secondary & 2 & 3 \\
\hline 33 & Retroperitoneum & High & Primary & 2 & 1 \\
\hline 34 & Retroperitoneum & High & Primary & 1 & 2 \\
\hline 35 & Thigh & High & Primary & 2 & 2 \\
\hline 36 & Retroperitoneum & High & Primary & 2 & 2 \\
\hline 37 & Retroperitoneum & High & Primary & 0 & 2 \\
\hline 38 & Retroperitoneum & Low & Primary & 3 & 2 \\
\hline 39 & Retroperitoneum & High & Primary & 1 & 2 \\
\hline 40 & Retroperitoneum & High & Primary & 3 & 0 \\
\hline
\end{tabular}

PPAR- $\gamma$, peroxisome proliferator gamma; WD, well-differentiated component; DD, dedifferentiated component. 

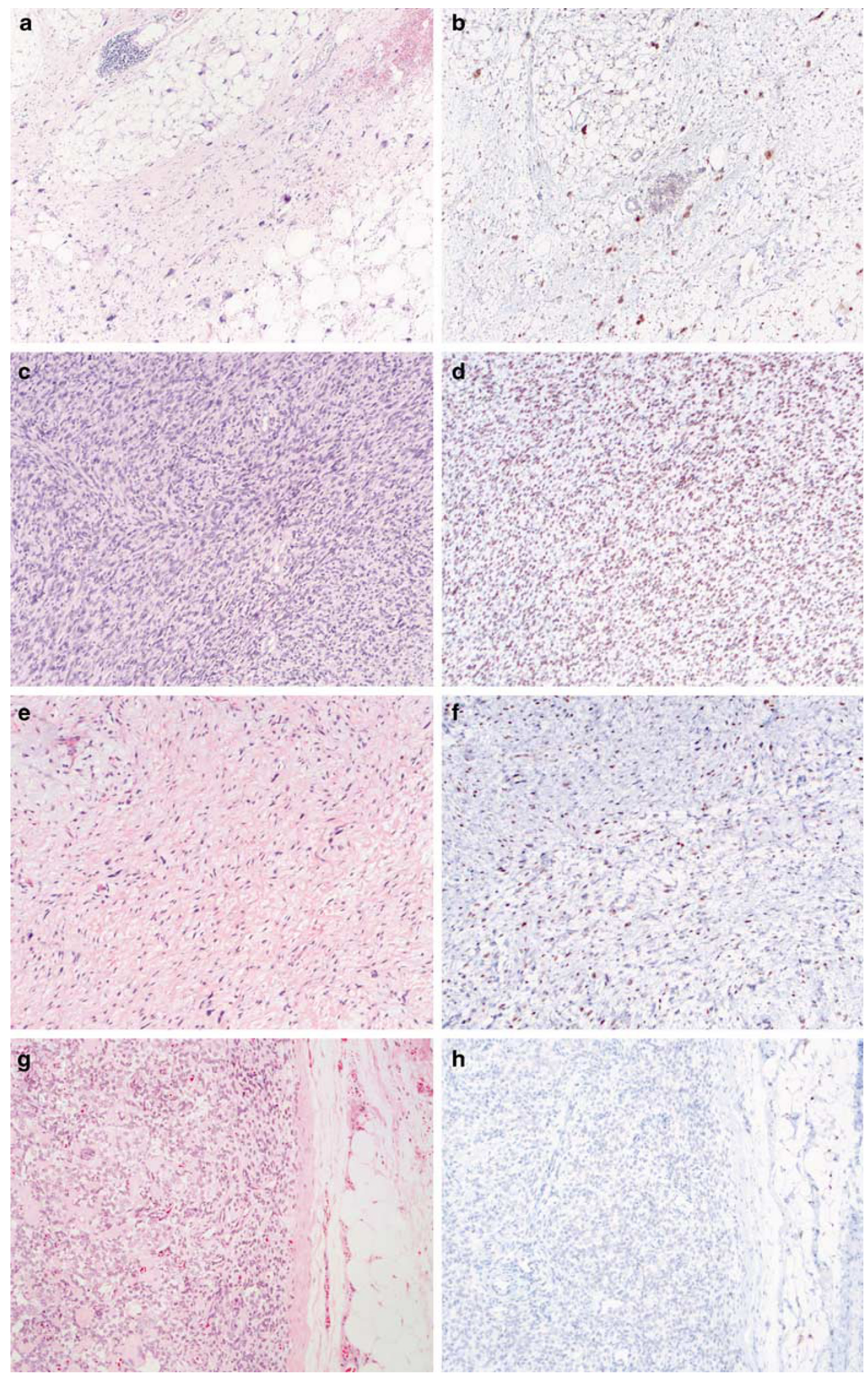

Figure 1 Representative histology and immunohistochemistry results comparing dedifferentiated liposarcomas and other retroperitoneal sarcomas. The left column demonstrates H\&E-stained slides, with corresponding immunohistochemistry for PPAR- $\gamma$ in the right column. Case 28 (a-d) demonstrates nuclear PPAR- $\gamma$ staining in the well-differentiated (b) and high-grade dedifferentiated (d) components. The dedifferentiated component of case 22 demonstrates low-grade histology (e) and is also PPAR- $\gamma$ positive (f). Case 14, an example of a nonlipogenic retroperitoneal sarcoma (g) that is negative for PPAR- $\gamma$ (note positive staining in adjacent benign adipose tissue) (h). 
$37 / 40(93 \%)$ dedifferentiated components of the dedifferentiated liposarcoma. Although the fraction of cells staining, and thus staining score, varied, only six cases $(15 \%)$ were discordant between the well-differentiated and dedifferentiated components for the presence of any PPAR- $\gamma$ immunostaining.

The comparison group included 24 retroperitoneal sarcomas, some of which also involved the kidney, mesentery, or small intestine, as summarized in Table 2. The most common diagnosis was undifferentiated sarcoma, including pleomorphic and spindle-cell predominant types (11/24, 46\%). Myxofibrosarcoma and leiomyosarcoma were equally common $(5 / 24,21 \%)$. Overall, PPAR- $\gamma$ immunostaining was observed in $6 / 24(25 \%)$ of the retroperitoneal sarcomas, of which four were undifferentiated sarcomas and two were leiomyosarcomas (Table 2). In some negative cases, benign adipose tissue adjacent to tumor (Figure $1 \mathrm{~g}$ ) stained with PPAR- $\gamma$ (Figure 1h) and served as an internal positive control. To better characterize the retroperitoneal sarcomas, we also evaluated immunohistochemistry for MDM2 and CDK4. None of the sarcomas with specific differentiation (leiomyosarcoma, malignant peripheral nerve sheath tumor, or endometrial stromal sarcoma) demonstrated any staining for these two markers. In contrast, nearly half of the sarcomas initially classified as undifferentiated and myxofibrosarcoma $(7 / 17,42 \%)$ were positive for either MDM2 or CDK4 with two cases $(12 \%)$ positive for both. Four of the six PPAR- $\gamma$ positive nonlipogenic sarcomas also showed nuclear staining with CDK4 or MDM2 or with both of these markers (Table 2).

\section{Discussion}

The diagnosis of mesenchymal neoplasms of the retroperitoneum is challenging, especially when the histologic diagnosis must be based on limited material such as that obtained by CT-guided biopsy. Unfortunately, the scant tissue from a minimally invasive biopsy may not sample the well-differentiated lipogenic component necessary for the diagnosis of dedifferentiated liposarcoma. Furthermore, some authors have suggested that a significant proportion of undifferentiated sarcomas of the retroperitoneum (so-called 'malignant fibrous histiocytoma') actually represent dedifferentiated liposarcoma. ${ }^{4,5,8}$ This study demonstrates that immunohistochemistry for the PPAR- $\gamma$ protein detects the vast majority of dedifferentiated liposarcomas in paraffin-embedded tissue sections. As might be predicted from the nuclear receptor function of PPAR- $\gamma$, the pattern of staining is exclusively nuclear. In contrast, a much smaller subset $(25 \%)$ of other retroperitoneal sarcomas demonstrates nuclear staining for PPAR- $\gamma$. Therefore, we propose that PPAR- $\gamma$ immunohistochemistry is a useful adjunct in the diagnosis of retroperitoneal tumors, in which dedifferentiated liposarcoma is often a consideration. PPAR- $\gamma$ appears to be a marker of adipocyte differentiation and is expressed in both

Table 2 Summary of clinical, pathologic, and immunohistochemical data for retroperitoneal sarcomas

\begin{tabular}{|c|c|c|c|c|}
\hline \multirow[t]{2}{*}{ Case } & \multirow[t]{2}{*}{ Diagnosis } & \multicolumn{3}{|c|}{ Immunohistochemistry } \\
\hline & & $P P A R-\gamma$ & $M D M 2$ & $C D K 4$ \\
\hline 1 & Myxofibrosarcoma & 0 & 0 & 0 \\
\hline 2 & Undifferentiated sarcoma, spindle cell & 0 & 3 & 0 \\
\hline 3 & Undifferentiated sarcoma, spindle cell & 1 & 0 & 1 \\
\hline 4 & Leiomyosarcoma & 0 & 0 & 0 \\
\hline 5 & Undifferentiated sarcoma, spindle cell & 0 & 0 & 0 \\
\hline 6 & Undifferentiated sarcoma, giant-cell rich & 0 & 0 & 0 \\
\hline 7 & Undifferentiated sarcoma, spindle cell & 0 & 0 & 0 \\
\hline 8 & Undifferentiated sarcoma, pleomorphic & 3 & 3 & 0 \\
\hline 9 & Leiomyosarcoma & 2 & 0 & 0 \\
\hline 10 & Leiomyosarcoma & 0 & 0 & 0 \\
\hline 11 & Leiomyosarcoma & 3 & 0 & 0 \\
\hline 12 & Malignant peripheral nerve sheath tumor & 0 & 0 & 0 \\
\hline 13 & Leiomyosarcoma & 0 & 0 & 0 \\
\hline 14 & Endometrial stromal sarcoma & 0 & 0 & 0 \\
\hline 15 & Undifferentiated sarcoma, pleomorphic & 4 & 2 & 1 \\
\hline 16 & Undifferentiated sarcoma, pleomorphic & 0 & 0 & 0 \\
\hline 17 & Myxofibrosarcoma & 0 & 0 & 0 \\
\hline 18 & Undifferentiated sarcoma, spindle cell & 3 & 3 & 0 \\
\hline 19 & Undifferentiated sarcoma, spindle cell & 0 & 0 & 0 \\
\hline 20 & Undifferentiated sarcoma, pleomorphic & 0 & 0 & 0 \\
\hline 21 & Undifferentiated sarcoma, pleomorphic & 0 & 0 & 0 \\
\hline 22 & Myxofibrosarcoma & 0 & 0 & 2 \\
\hline 23 & Myxofibrosarcoma & 0 & 0 & 0 \\
\hline 24 & Myxofibrosarcoma & 0 & 1 & 1 \\
\hline
\end{tabular}

PPAR- $\gamma$, peroxisome proliferator gamma. 
benign adipose tissue and liposarcoma. Thus PPAR- $\gamma$ staining cannot separate benign from malignant adipose tumors. However, immunohistochemical detection of this protein in a sarcoma can suggest lipogenic differentiation when such differentiation is not histologically obvious.

Recent work by Binh et $a l^{8}$ has shown that immunohistochemistry for the proteins MDM2 and CDK4 is useful to distinguish between lipomas and liposarcomas. The overexpression of these proteins appears to stem from amplification of the corresponding genes on the chromosomal region 12q14-12q15 in liposarcomas, but not lipomas. ${ }^{23,24}$ Although the exact role of $C d k 4$ and $M d m 2$ gene amplification in liposarcoma tumorigenesis is not known, abnormal expression of these gene products may lead to loss of cell-cycle control. Given that dysregulation of cell-cycle machinery has variable tumor specificity, it follows that elevated levels of CDK4 and MDM2 proteins can be detected by immunohistochemistry in a number of sarcoma types. $^{9-11,25-27}$ Interestingly, however, recent reports suggest that these markers are also useful to distinguish some poorly differentiated sarcomas from dedifferentiated liposarcoma. ${ }^{7,8} \mathrm{We}$ recognize that the histologic evidence of a well-differentiated component is the so-called 'gold standard' for the diagnosis of dedifferentiated liposarcoma. However, we sought to better characterize the group of retroperitoneal sarcomas in our series with respect to CDK4 and MDM2 expression, first to determine the relationship of these markers to PPAR- $\gamma$ and, second, to explore the possibility that some of these tumors represent dedifferentiated liposarcoma (Table 2). Intriguingly, we noted that four of the six PPAR- $\gamma$ positive nonlipogenic sarcomas also showed nuclear staining with CDK4 or MDM2 or with both of these markers. Although none of these cases demonstrated a component of well-differentiated liposarcoma on H\&E sections, we cannot completely exclude that some of these cases represent dedifferentiated liposarcoma in which the well-differentiated component was either inadequately sampled or completely replaced by the nonlipogenic component. Although the CDK4-and MDM2-positive immunophenotype certainly does not equate to an unequivocal diagnosis of dedifferentiated liposarcoma, if CDK4- and MDM2-positive cases do represent liposarcomas, these data further support the specificity of PPAR- $\gamma$ in separating dedifferentiated liposarcoma from other retroperitoneal sarcomas.

Clinical outcome in dedifferentiated liposarcoma appears to be independent of tumor grade., ${ }^{3,28}$ However, this observation is based on a relatively small number of patients, and since most cases arise in the retroperitoneum, local tumor effects, rather than metastasis, often influence the outcome. Insofar as PPAR- $\gamma$ mediates terminal differentiation of the adipocyte lineage, ${ }^{12,13,29}$ absent or decreased PPAR- $\gamma$ expression may be predicted in the dedifferentiated component. However, in this study, the well-differentiated and dedifferentiated components of dedifferentiated liposarcoma stained in an approximately equal proportion of cases. Although the concordance between pairs of well-differentiated and dedifferentiated areas was not absolute, we observed no definite trend for loss of PPAR- $\gamma$ staining in the latter regions. Interestingly, all the three cases of dedifferentiated liposarcoma with negative PPAR- $\gamma$ immunohistochemistry shared high-grade histology, whereas all cases with lowgrade histology retained PPAR- $\gamma$, suggesting that higher histologic grade may indicate some deficiency in adipocyte differentiation mechanisms. We acknowledge, however, that the number of cases in these groups is too small to draw specific conclusions about a progression model.

The PPAR- $\gamma$ nuclear receptor regulates growth and differentiation of adipocytes and has been explored as a molecular target for therapy. Data from in vitro studies initially suggested that PPAR- $\gamma$ may be a useful target to induce differentiation and withdrawal from the cell cycle of liposarcoma cells. ${ }^{15}$ To date, clinical trials with the thiazolidinedione family of drugs have shown mixed results with respect to efficacy in the treatment of liposarcomas. ${ }^{16,17}$ However, these studies included myxoid-round cell and pleomorphic liposarcomas and only two patients with dedifferentiated liposarcoma. Furthermore, the only consistent induction of PPAR- $\gamma$ responsive genes during therapy was in one of the dedifferentiated liposarcoma tumors. ${ }^{17}$ Subsequent studies suggested that mechanisms independent of PPAR- $\gamma$ activation mediated the antitumor effects of these drugs and stimulated more widespread interest in thiazolidinediones as cancer therapy. ${ }^{30-32}$ Consequently, the efficacy of PPAR- $\gamma$ ligands on a variety of nonlipogenic malignancies was tested but, unfortunately, yielded little improvement in outcome. ${ }^{33,34}$ Thus, enthusiasm for the use of PPAR- $\gamma$ ligands as differentiation-promoting therapy has somewhat waned. ${ }^{30}$ Nevertheless, our study suggests that the vast majority of dedifferentiated liposarcoma retain detectable levels of PPAR- $\gamma$ protein, and re-evaluation of this selected group of sarcomas with therapy targeting PPAR- $\gamma$ may be worthwhile.

In summary, immunohistochemistry for PPAR- $\gamma$ is a convenient and rapid technique for the evaluation of sarcomas, including histologically low-grade examples. Especially in a small biopsy of a retroperitoneal sarcoma with uncertain histogenesis, positive PPAR- $\gamma$ staining is a useful adjunct to routine histology. In combination with other markers, PPAR- $\gamma$ staining can help establish a diagnosis of dedifferentiated liposarcoma.

\section{Acknowledgement}

We thank the Immunopathology Laboratories at UCSF and SFGH for their technical assistance. 


\section{Disclosure/conflict of interest}

None declared.

\section{References}

1 Fletcher CDM, Unni KK, Mertens F (eds). Pathology and Genetics of Tumours of Soft Tissue and Bone. IARC Press: Lyon, 2002.

2 Weiss SW, Goldblum JR. Enzinger and Weiss's Soft Tissue Tumors. Mosby: St Louis, 2001.

3 Henricks WH, Chu YC, Goldblum JR, et al. Dedifferentiated liposarcoma: a clinicopathological analysis of 155 cases with a proposal for an expanded definition of dedifferentiation. Am J Surg Pathol 1997;21:271-281.

4 Coindre JM, Mariani O, Chibon F, et al. Most malignant fibrous histiocytomas developed in the retroperitoneum are dedifferentiated liposarcomas: a review of 25 cases initially diagnosed as malignant fibrous histiocytoma. Mod Pathol 2003;16:256-262.

5 Coindre JM, Hostein I, Maire G, et al. Inflammatory malignant fibrous histiocytomas and dedifferentiated liposarcomas: histological review, genomic profile, and MDM2 and CDK4 status favour a single entity. J Pathol 2004;203:822-830.

6 McCormick D, Mentzel T, Beham A, et al. Dedifferentiated liposarcoma. Clinicopathologic analysis of 32 cases suggesting a better prognostic subgroup among pleomorphic sarcomas. Am J Surg Pathol 1994;18: 1213-1223.

7 Binh MB, Garau XS, Guillou L, et al. Reproducibility of MDM2 and CDK4 staining in soft tissue tumors. Am J Clin Pathol 2006;125:693-697.

8 Binh MB, Sastre-Garau X, Guillou L, et al. MDM2 and CDK4 immunostainings are useful adjuncts in diagnosing well-differentiated and dedifferentiated liposarcoma subtypes: a comparative analysis of 559 soft tissue neoplasms with genetic data. Am J Surg Pathol 2005;29:1340-1347.

9 Momand J, Jung D, Wilczynski S, et al. The MDM2 gene amplification database. Nucleic Acids Res 1998;26:3453-3459.

10 Florenes VA, Maelandsmo GM, Forus A, et al. MDM2 gene amplification and transcript levels in human sarcomas: relationship to TP53 gene status. J Natl Cancer Inst 1994;86:1297-1302.

11 Ladanyi M, Lewis R, Jhanwar SC, et al. MDM2 and CDK4 gene amplification in Ewing's sarcoma. J Pathol 1995;175:211-217.

12 Tontonoz P, Hu E, Graves RA, et al. mPPAR gamma 2: tissue-specific regulator of an adipocyte enhancer. Genes Dev 1994;8:1224-1234.

13 Tontonoz P, Graves RA, Budavari AI, et al. Adipocytespecific transcription factor ARF6 is a heterodimeric complex of two nuclear hormone receptors, PPAR gamma and RXR alpha. Nucleic Acids Res 1994;22: 5628-5634.

14 Tontonoz P, Hu E, Spiegelman BM. Regulation of adipocyte gene expression and differentiation by peroxisome proliferator activated receptor gamma. Curr Opin Genet Dev 1995;5:571-576.

15 Tontonoz P, Singer S, Forman BM, et al. Terminal differentiation of human liposarcoma cells induced by ligands for peroxisome proliferator-activated receptor gamma and the retinoid X receptor. Proc Natl Acad Sci USA 1997;94:237-241.

16 Demetri GD, Fletcher CD, Mueller E, et al. Induction of solid tumor differentiation by the peroxisome proliferator-activated receptor-gamma ligand troglitazone in patients with liposarcoma. Proc Natl Acad Sci USA 1999;96:3951-3956.

17 Debrock G, Vanhentenrijk V, Sciot R, et al. A phase II trial with rosiglitazone in liposarcoma patients. Br J Cancer 2003;89:1409-1412.

18 Weiss SW, Rao VK. Well-differentiated liposarcoma (atypical lipoma) of deep soft tissue of the extremities, retroperitoneum, and miscellaneous sites. A follow-up study of 92 cases with analysis of the incidence of 'dedifferentiation'. Am J Surg Pathol 1992;16: 1051-1058.

19 Horvai AE, Kramer MJ, O’Donnell R. Beta-catenin nuclear expression correlates with cyclin D1 expression in primary and metastatic synovial sarcoma: a tissue microarray study. Arch Pathol Lab Med 2006; 130:792-798.

20 Horvai AE, Li L, Xu Z, et al. c-Kit is not expressed in malignant mesothelioma. Mod Pathol 2003;16: 818-822.

21 Fanburg-Smith JC, Miettinen M. Liposarcoma with meningothelial-like whorls: a study of 17 cases of a distinctive histological pattern associated with dedifferentiated liposarcoma. Histopathology 1998;33: 414-424.

22 Nascimento AG, Kurtin PJ, Guillou L, et al. Dedifferentiated liposarcoma: a report of nine cases with a peculiar neurallike whorling pattern associated with metaplastic bone formation. Am J Surg Pathol 1998;22:945-955.

23 Dal Cin P, Kools P, Sciot R, et al. Cytogenetic and fluorescence in situ hybridization investigation of ring chromosomes characterizing a specific pathologic subgroup of adipose tissue tumors. Cancer Genet Cytogenet 1993;68:85-90.

24 Pedeutour F, Suijkerbuijk RF, Van Gaal J, et al. Chromosome 12 origin in rings and giant markers in well-differentiated liposarcoma. Cancer Genet Cytogenet 1993;66:133-134.

25 Takahashi Y, Oda Y, Kawaguchi K, et al. Altered expression and molecular abnormalities of cell-cycleregulatory proteins in rhabdomyosarcoma. Mod Pathol 2004;17:660-669.

26 Oda Y, Sakamoto A, Satio T, et al. Molecular abnormalities of p53, MDM2, and H-ras in synovial sarcoma. Mod Pathol 2000;13:994-1004.

27 Sabah M, Cummins R, Leader $\mathrm{M}$, et al. Immunoreactivity of p53, Mdm2, p21(WAF1/CIP1) Bcl-2, and Bax in soft tissue sarcomas: correlation with histologic grade. Appl Immunohistochem Mol Morphol 2007;15: 64-69.

28 Elgar F, Goldblum JR. Well-differentiated liposarcoma of the retroperitoneum: a clinicopathologic analysis of 20 cases, with particular attention to the extent of low-grade dedifferentiation. Mod Pathol 1997;10:113-120.

29 Kliewer SA, Forman BM, Blumberg B, et al. Differential expression and activation of a family of murine peroxisome proliferator-activated receptors. Proc Natl Acad Sci USA 1994;91:7355-7359.

30 Rumi MA, Ishihara S, Kazumori H, et al. Can PPAR gamma ligands be used in cancer therapy? Curr Med Chem Anticancer Agents 2004;4:465-477. 
31 Palakurthi SS, Aktas H, Grubissich LM, et al. Anticancer effects of thiazolidinediones are independent of peroxisome proliferator-activated receptor gamma and mediated by inhibition of translation initiation. Cancer Res 2001;61:6213-6218.

32 Panigrahy D, Shen LQ, Kieran MW, et al. Therapeutic potential of thiazolidinediones as anticancer agents. Expert Opin Investig Drugs 2003;12: 1925-1937.
33 Kulke MH, Demetri GD, Sharpless NE, et al. A phase II study of troglitazone, an activator of the PPARgamma receptor, in patients with chemotherapy-resistant metastatic colorectal cancer. Cancer J 2002;8:395-399.

34 Burstein HJ, Demetri GD, Mueller E, et al. Use of the peroxisome proliferator-activated receptor (PPAR) gamma ligand troglitazone as treatment for refractory breast cancer: a phase II study. Breast Cancer Res Treat 2003;79:391-397. 